\title{
Are Kinesiophobia and Functional Performance Related to Ability to Forget the Artificial Joint in Patients with Total Hip Arthroplasty?
}

\author{
Aykut Ozcadirci $^{1}$, Ferhat Ozturk ${ }^{1}$, Omur Caglar ${ }^{2}$, Gursoy Coskun ${ }^{1}$ \\ ${ }^{1}$ Faculty of Physical Therapy and Rehabilitation, Hacettepe University, Ankara, Turkey \\ ${ }^{2}$ Department of Orthopaedics and Traumatology, Faculty of Medicine, Hacettepe University, Ankara, Turkey
}

Correspondence Author: Aykut Ozcadirci

E-mail: aykutozcadirci@gmail.com

Received: $26.03 .2021 \quad$ Accepted: 22.05 .2021

\begin{abstract}
Objective: The aim of this study was to investigate the relationship between kinesiophobia and functional performance with the ability to forgetthe artificial joint in patients with total hip arthroplasty (THA).

Methods: Fifty-seven volunteer patients aged between 40-65 years who have undergone THA surgery in the last 1-3 years were included in this study. The Forgotten Joint Score (FJS-12) for evaluating the ability to forgetting the artificial joint, the 6-Minute Walk Test for determining the functional performance, and the Tampa Scale for Kinesiophobia (TSK) for evaluating kinesiophobia were used.

Results: There was a moderate correlation between the FJS-12 and TSK ( $r=-0.571 ; p<0.001)$ scores; and moderate correlation between the FJS-12 and the 6-Minute Walk distances (6-MWD) $(r=0.408 ; p<0.001)$. The ability to forget the artificial joint in patients with THA was found to be related to kinesiophobia and functional performance.

Conclusion: The results of this study demonstrated that the awareness of artificial joint should be taken into consideration when performing physiotherapy and rehabilitation practices to decrease kinesiophobia and increase functional performance. Additionally, complementary approaches aiming to reduce the awareness of artificial joint should be added to the rehabilitation program of the patients with total hip arthroplasty.
\end{abstract}

Keywords: Arthroplasty, awareness, functional performance, hip.

\section{INTRODUCTION}

Total Hip Arthroplasty (THA) is one of the most commonly used procedure in the late treatment of hip osteoarthritis (1). Arthroplasty surgery, which aims to reduce pain and improve physical function, enables the patient to participate in daily living activities and return to an active lifestyle $(2,3)$. Patients undergoing THA surgery complain of pain and loss of motion before surgery. Therefore, THA surgery outcome measures are more related to decreased pain complaints and increased mobility. Although the functional status is good after surgery, patients with THA have less participation in daily living activities. One of the reasons for this is maybe that the patients develop artificial joint awareness and cannot forget the new joint $(4,5)$.

Conventional classification systems that evaluate outcomes after TKA often focuses on objective parameters such as range of motion and strength, or clinician ratings and degrees of pain. However, expectations of patients after TKA may different importantly from the expectations of clinicians and may not be sufficient to reflect the needs and views of patients $(6-8)$. It is thought that the expectations of approximately $20-30 \%$ of patients after joint arthroplasty surgery are not fully satisfied expectations (7). Therefore, patient-reported outcome measures, including patient-specific or jointspecific parameters such as pain, the function of daily living activities and joint awareness, are increasingly recognized as an important part of postoperative outcome assessment $(6,9)$. Patient-reported outcomes (PRO) measures which providing a patient-centered view of treatment evaluation are a key parameter for evaluating outcomes after THA surgery (10-12).

Kinesiophobia is a poor adaptation strategy that causes patients to avoid physical activity due to fears related to pain (13). It has been reported that the incidence of kinesiophobia in orthopedic trauma patients may be as high as $52.8 \%$ (14). Doury-Panchout et al. (15), in a study investigating the effect of kinesiophobia on healing after surgery, was shown that 
the presence of kinesiophobia in total knee arthroplasty (TKA) had a negative effect on healing.

THA surgery is a surgical procedure that usually results in a significant decrease in pain and improvements in patients' functional capacity. However, despite the decrease in pain on the operated side, it has been reported that in many cases, motor skills do not reach normal levels (16-18). The ability to walk, which is related to leading an active and independent life, is an important component of lower extremity function. Evaluation of functional performance after THA provides important information about the healing process. Some studies have shown that patients feel less pain and walk better than preoperative 3 months after THA (19, 20). However, further studies have shown that the walking distance of patients months and years after THA is less than that of healthy individuals of the same age, hip flexibility and muscle strength are worse than unaffected hips $(17,21)$.

In the literature, there are studies comparing and evaluating the functional capacity of patients before and after THA (2224). However, in the literature, there is no study to investigate the relationship between kinesiophobia and functional performance with the ability to forget the artificial joint in patients with THA. It is not known whether the patient's fear of movement and functional performance are relateds to the artificial joint awareness. Therefore, the aim of this study was to investigate the relationship between kinesiophobia and functional performance with the ability to forget the artificial joint in patients with THA. The authors hypothesized that the ability to forget the artificial joint would be associated with kinesiophobia and functional performance in patients with THA.

\section{METHODS}

In this study, 57 patients who had undergone THA surgery between the ages of 40-65 yearsat least 1 and at most 3 years were included. The study was approved by the Hacettepe University Ethics Committee for Non-Interventional Clinical Investigations (GO17 / 878-31). Before starting the study, volunteered patients were informed verbally about the purpose of the study, the duration of the assessment. Patients signed an informed consent form that they were willing to participate in the study. Patients who have undergone lower extremity surgery other than THA, signs of infection and have a history of THA revision or dislocation were excluded from the study.

\subsection{Assessment of the Ability to Forget the Artificial Joint}

The FJS-12 was used to evaluate the ability to forget artificial joint of patients with THA. The FJS-12 is a scale that questions the artificial joint awareness during various daily living activities in order to determine the ability of patients to forget the artificial hip joint(25). A validated Turkish version of the FJS-12 was used in this study (26). A 5-point Likert system is used in the scoring (None: 0, Almost none: 1, Rarely: 2, Sometimes: 3, Always: 4). All answers to the questionnaire $(0 ; 1 ; 2 ; 3 ; 4)$ are summed and divided by the number of completed questions. The calculated average value is multiplied by 25 so that the total score is in the range $0-100$. The number found is subtracted from 100 . High scores indicate how much (\%) the patient can forget their side of the surgery and adapt to their life. In other words, a high score indicates a high degree of "forgetting" the artificial joint - i.e. a low degree of awareness(26).

\subsection{Assessment of Kinesiophobia}

The Tampa Scale for Kinesiophobia (TSK) was used to evaluate the presence of kinesiophobia . The TSK is a 17item scale developed to measure kinesiophobia / re-injury. The kinesiophobia of the patients included in this study was measured with the Turkish TSK (27). The scale uses a 4-point Likert rating (Strongly disagree: 1, Disagree: 2, Agree: 3, Completely agree: 4$)$. A total score is calculated by reversing questions 4, 8, 12 and 16 . Patients have a total score of from 17 to 68 . The high score of the person on the scale shows that kinesiophobia is also high (28).

\subsection{Assessment of Functional Performance}

In order to determine the functional performance of the patients, 6-MWD was performed. This test which is one of the tests used to evaluate functional performance in patients with THA is a simple, sensitive and valid method used in the clinic (29). The distance to walk according to age, sex, height and body weight is calculated (30). For each patient, the distance walking is calculated in meters and as a percentage of the theoretical distance. Patients were asked to walk on a flat surface for 6 minutes. During the test, standard expressions were used to the patients during their gait. For each patient, the distance walking was calculated and recorded in "meters" and as a percentage of the theoretical distance (expected walking distance) (Figure 1).

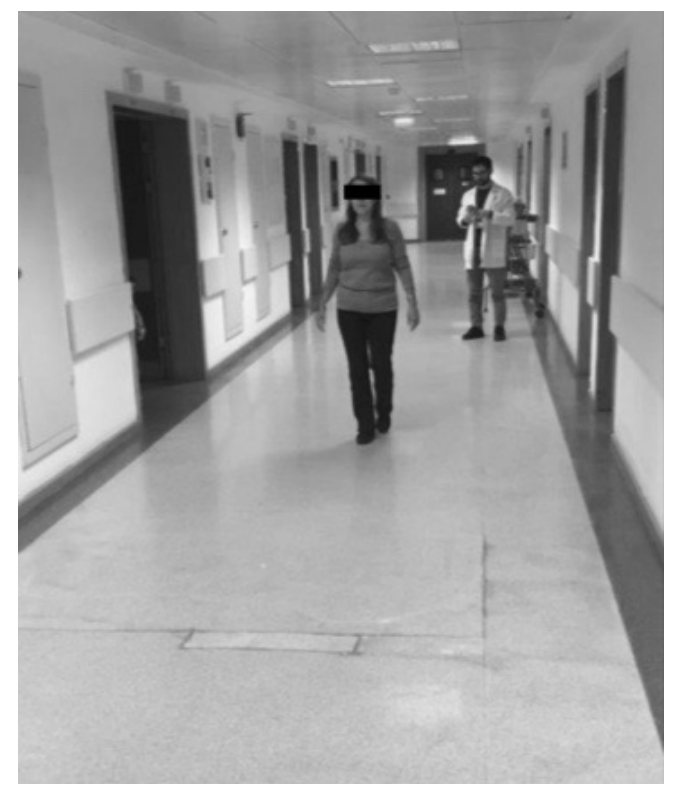

Figure 1. Six minute walking distance 


\subsection{Statistical Analysis}

The data obtained were evaluated with SPSS 23.0 software program (IBM SPSS Statistics version 23.0, IBM Corp. Armonk, New York, ABD). The normal distribution of the variables was determined by visual (histogram and probability graphs) and analytical methods (Kolmogorov-Smirnov / Shapiro-Wilk tests). Descriptive analyzes mean and standard deviation for numerical variables; frequency tables for ordinal variables (n) and proportions (\%) are shown.

Mann-Whitney $U$ test was used to determine whether FJS, TSK scores, and 6MWD values differ by gender. Kruskal Wallis analysis was used to compare the joint awareness, kinesiophobia, and functional performance according to the preoperative diagnosis. In addition, Spearman Correlation Analysis was used for the variables that do not show normal distribution to the determine relationship between the ability to forget the artificial joint, kinesiophobia, functional performance.

\section{RESULTS}

A total of 57 patients (12 males; 45 females) were evaluated in this study. Demographic characteristics of patients such as educational status, preoperative diagnosis, job and mean BMI values are summarized in Table 1.

Table 1. Sociodemographic characteristics of the patients.

\begin{tabular}{|c|c|}
\hline & Mean (SD, Minimum-Maximum) \\
\hline \multicolumn{2}{|l|}{ Gender, n (\%) } \\
\hline \multirow{2}{*}{$\begin{array}{l}\text { Female } \\
\text { Male }\end{array}$} & 45 (78.9\%) \\
\hline & $12(21.1 \%)$ \\
\hline Age & $55.39(7.8,40-65)$ \\
\hline Height $(\mathrm{cm})$ & $163.19(9.5,145-182)$ \\
\hline Weight (kg) & $75.63(14.3,50-120)$ \\
\hline Body mass index $\left(\mathrm{kg} / \mathrm{m}^{2}\right)$ & $28.25(4.6,20.3-44.19)$ \\
\hline Duration after surgery (months) & $24.78(4.6,12-36)$ \\
\hline \multicolumn{2}{|l|}{ Diagnosis, $\mathrm{n}(\%)$} \\
\hline \multirow{4}{*}{$\begin{array}{l}\text { Primary osteoarthritis } \\
\text { Avascular necrosis } \\
\text { Developmental dysplasia of the hip } \\
\text { Fracture of femur }\end{array}$} & $30(52.6 \%)$ \\
\hline & $12(21.1 \%)$ \\
\hline & $11(19.3 \%)$ \\
\hline & $4(7 \%)$ \\
\hline \multicolumn{2}{|l|}{ Education status $\mathrm{n}(\%)$} \\
\hline Primary school & $28(49.1 \%)$ \\
\hline Middle school & $5(8.8 \%)$ \\
\hline \multirow{2}{*}{ High school } & $11(19.3 \%)$ \\
\hline & $13(22.8 \%)$ \\
\hline \multicolumn{2}{|l|}{ Occupational status $\mathrm{n}(\%)$} \\
\hline \multirow{3}{*}{$\begin{array}{l}\text { Housewife } \\
\text { Working } \\
\text { Retired }\end{array}$} & $24(42.1 \%)$ \\
\hline & $17(29.8 \%)$ \\
\hline & $16(28.1 \%)$ \\
\hline \multicolumn{2}{|l|}{ Patient reported outcomes } \\
\hline \multirow{2}{*}{$\begin{array}{l}\text { Forgetten Joint Score-12 } \\
\text { Tampa Scale Kinesiophobia }\end{array}$} & $31.25^{*}\left(35.45^{\dagger}, 0-100\right)$ \\
\hline & $46.0^{*}\left(10^{\dagger}, 27-64\right)$ \\
\hline
\end{tabular}

SD: Standard Deviation, THA: Total Hip Arthroplasty, ${ }^{*}$ : Median, +. Interquartile Range (IQR)
The minimum, maximum, median and Interquartile range (IQR) values of the FJS-12, TSK scores are shown in Table 1.

No statistically significant difference was observed in the distribution of the FJS-12, TSK scores, and 6-MWD of the patients according to gender (respectively $p=0.638, p=0.462$, $\mathrm{p}=0.610)$.

The comparison of joint awareness, kinesiophobia, and functional performance results according to the preoperative diagnosis is summarized in Table 2. Kruskal Wallis analysis showed that the results were similar according to the preoperative diagnosis.

Table 2. Comparison of FJS, TSK, and 6-MWD according to preoperative diagnosis

\begin{tabular}{|c|c|c|c|c|}
\hline & Preoperative diagnosis & $n$ & $\chi^{2}$ & $\mathrm{p}$ \\
\hline \multirow{4}{*}{ FJS-12 } & Primary osteoarthritis & 30 & \multirow{4}{*}{2.04} & \multirow{4}{*}{0.563} \\
\hline & Avascular necrosis & 11 & & \\
\hline & Developmental dysplasia of the hip & 4 & & \\
\hline & Fracture of femur & 12 & & \\
\hline \multirow{4}{*}{ TSK } & Primary osteoarthritis & 30 & \multirow{4}{*}{0.13} & \multirow{4}{*}{0.988} \\
\hline & Avascular necrosis & 11 & & \\
\hline & Developmental dysplasia of the hip & 4 & & \\
\hline & Fracture of femur & 12 & & \\
\hline \multirow{4}{*}{ 6-MWD } & Primary osteoarthritis & 30 & \multirow{4}{*}{3.99} & \multirow{4}{*}{0.262} \\
\hline & Avascular necrosis & 11 & & \\
\hline & Developmental dysplasia of the hip & 4 & & \\
\hline & Fracture of femur & 12 & & \\
\hline
\end{tabular}

FJS-12: Forgotten Joint Score, TSK: Tampa Scale for Kinesiophobia, 6-MWD: 6-Minute Walk Distance, $\chi^{2}$ : Chi-Square,

The mean and minimum-maximum values of 6-MWD, which are theoretically calculated for patients (expected walking distance) and are walking distance during the test, are shown in Table 3. All of the patients included in the study were able to walk less than the expected walking distance. In addition, it was found that $68.08 \%$ of the theoretical distance calculated in 6-MWD was able to walk.

Table 3. Walking distance and expected distance in 6-MWD.

\begin{tabular}{|c|c|c|c|c|}
\hline $\mathrm{n}=57$ & \multicolumn{2}{|c|}{ Walking Distance $(\mathrm{m})$} & \multicolumn{2}{c|}{ Expected Distance $(\mathrm{m})$} \\
\hline \multirow{2}{*}{ 6-MWD } & Mean (SD) & $\begin{array}{c}\text { Minimum- } \\
\text { Maximum }\end{array}$ & Mean (SD) & $\begin{array}{c}\text { Minimum- } \\
\text { Maximum }\end{array}$ \\
\cline { 2 - 5 } & $357.49 \pm 55$ & $231-483$ & $529.48 \pm 61$ & $370.32-745.20$ \\
\hline
\end{tabular}

6-MWD: 6-Minute Walk Distance, 6-MWT: 6 Minute Walking Test, SD: Standard Deviation

The Spearman correlation analysis showed that there was a moderate negative correlation between the FJS-12 and TSK $(r=-0.571 ; p<0.001)$; and moderate correlation between the FJS-12 and 6-MWD ( $r=0.408 ; p<0.001)$ (Table 4).

Table 4. Correlation analysis between FJS-12, TSK and 6-MWD

\begin{tabular}{|c|c|c|c|}
\hline \multicolumn{2}{|c|}{$n=57$} & $r$ & $p$ \\
\hline \multirow{2}{*}{ FJS-12 } & TSK & -0.571 & $<0.001$ \\
\cline { 2 - 4 } & $6-M W D$ & 0.408 & $<0.001$ \\
\hline
\end{tabular}

r: Spearman Correlation Coefficient, FJS-12: Forgotten Joint Score, TSK: Tampa Scale for Kinesiophobia, 6-MWD: 6-Minute Walk Distance 


\section{DISCUSSION}

This study is an important study in terms of investigating the relationship between the ability to forget the artificial joint and kinesophobia and functional performance in patients with THA. The main results of this study showed that the ability to forget the artificial joint in patients with THA is correlated with kinesiophobia and functional performance. The second important result of this study is that kinesiophobia is still high in patients even in the postoperative second year after surgery, functional performance is not at the expected level, and the ability to forget the artificial joint, ie adaptation to $A D L$, is insufficient.

Patient-reported outcome measures (PRO) are a key parameter to evaluate outcomes after THA surgery (12). The FJS-12 is a patient-reported outcome scale developed to evaluate joint awareness in the hip and knee joints during various activities in daily life that gives information about how much patients can forget their artificial joints and adapt to daily life (4). Unlike other outcome measures reported by the patient, which measures parameters such as pain, stiffness, difficulties in daily living activities, the FJS-12 emphasizes "awareness". In this study, the FJS-12 scale was used to evaluate the ability to forget the artificial joint.

Hamilton et al. (4) were reported the FJS-12 scores as 56.8 and 62.1 points 6 and 12 months after THA surgery. In another study, Thienpont et al. (31) stated that the FJS-12 score was 70 points for patients with THA in the 12th postoperative month of 75 patients (mean age 67) whom they evaluated before and after THA and TKA surgery. Similarly, Matsumoto et al. (32) were stated the FJS-12 scores as 61.1 in healthy individuals, 55.2 in OA patients and 45.8 in patients with THA in the first year after surgery.

The mean FJS-12 scores in this study was 36.58 . Unlike the studies in the literature $(4,31,32)$, the FJS-12 scores of the patients in the current study were low. In other words, low degree of forgetting their artificial joint was found in our patients after average 24 months following THA surgery. These results showed that our patients had still difficulty in adapting their new artificial joints during daily life. One possible explanation of this low score migth be high kinesiophobia. In addition, during the assessment patients verbally stated that they experienced fear of falling and this fear brought the prosthesis into their minds during their daily life activities. Therefore one of the reasons for high FJS-12 scores may be fear of falling in patients. Fear of dislocation may also be the reason to focus their attention on their artificial prosthesis during daily living activities. Another reason of high FJS-12 scores might be the lower rate of returning to work after surgery. Since the majority of the patients in the current study were housewives $(42.1 \%)$ or retired (28.1\%). Therefore, the limited daily living activities and low educational status ( $80 \%$ under bachelor degree) might canalize their attention on their artificial prostheses.

Kinesiophobia is described as the irrational and debilitating fear of physical movement and activity resulting from a sense of fragility caused by painful injury or re-injury (33). In the literature, studies evaluating the kinesiophobia of patients after THA surgery are limited. Sengül et al. (34), in their study that evaluated the fear of pain-related movement in patients with 58 patients who underwent prosthesis surgery (at least 2 years after surgery) for hip fracture or $O A$, found that the scores of the TSK were higher in both groups and that there was a high-strength relationship between the TSK and the Harris Hip Score.

In the postoperative period, kinesiophobia develops and patients are reluctant to move. As a result of their studies, it has been shown that even if they have high physical activity levels, the fear of moving in the postoperative period continues. As a matter of fact, according to one of the results of the current study, there was a statistically significant correlation between the FJS-12 and TSK scores of patients after THA surgery (at average 24 months). The fact that patients have been aware of the presence of artificial joints even long after their surgery showed that they had still high kinesiophobia.

Padovan et al (35) examined the effects of a new holistic approach on pain, fear of motion and quality of life in patients who underwent total hip arthroplasty, have evaluated the kinesiophobia with the Tampa Scale for Kinesiophobia and have found the experimental group's baseline TSK score as 30.6 and the control group as 36.53. Temporiti et al (36) examined the gait analysis after bilateral and unilateral total hip arthroplasty, and found that the TSK score of patients with unilateral arthroplasty was 36.4 , and that of patients with bilateral arthroplasty was 34.1. In contrast to this study, the TSK scores of the patients with TKA in our study were higher. This indicates that the kinesiophobia is still higher in our sample. The high kinesophobia scores may be due to the high artificial joint awareness. Correlation between artificial joint awareness and kinesiophobia scores supports this situation. In addition, the fear of dislocation may have contributed to the high rate of kinesophobia.

One of the outcome measures of THA surgery is the improvement of function. Additionally, one of the goals after THA surgery is to provide normal speed and adequate gait since gait is an important function of the lower extremity. Improvement of the physical function is evaluated by objective performance tests such as 6-MWD $(37,38)$. Evaluation of gait after THA surgery indicates functional performance in THA patients.

De Groot et al. (22) evaluated the walking capacities of patients by using 6-MWD at different periods after TKA and THA surgery, and found that the walking distance of the patients was $370 \mathrm{~m}$ at $3 \mathrm{rd}$ months, and $399 \mathrm{~m}$ at 6th months after surgery. Similarly, Heiberg et al. (39), the physical function by using 6MWD during the first year after THA surgery, and found that the walking distance of the patients was $401 \mathrm{~m}$ before surgery, $437 \mathrm{~m}$ at the 3rd month and 512 $\mathrm{m}$ at the 12 th postoperative month. 
Laupacis et al. (24), in their studies examining health-related quality of life after THA surgery, evaluated patients before and after surgery and stated that there was a considerable recovery in all health-related quality of life measurements and 6-MWDafter surgery. In two years, the average 6-MWD increased from $247 \mathrm{~m}$ to $408 \mathrm{~m}$. In the present study, the average 6-MWD of the patients was 357.49 meters. All the patients whose walking distance were calculated according to variables such as age, height and weight remained below the expected walking distance. The patients with THA walked only $68.08 \%$ of the calculated expected walking distance, theoretically. This study examined the long-term results of THA surgery and showed that patients' functional performance was still low. Parameters such as kinesiophobia, avoidance movement to protect prosthesis and high artificial joint awareness might have contributed to low functional performance results in 6-MWD.In addition, there was a moderate relationship between the ability to forget the artificial joint and functional performance in patients with THA. These results showed that the patients' functional performance would be higher as they could forget their artificial joints to adapt to daily life.

In this study, preoperative diagnosis status did not affect the results. The joint awareness and kinesiophobia level and functional performance of the patients with THA were similar between preoperative diagnosis. Not showing a homogeneous distribution of preoperative diagnosis may have caused these results.

The current study has some limitations. The most important limitation of our study is the absence of preoperative 6-MWD data of individuals with THA. Additionally, small sample size and cross-sectional design of the present study might be insufficient to generalize the results. Thus, pre-post follow-up studies involving larger sample sizes are needed. Future studies should also focus on parameters such as balance, fear of falling, quality of life, anxiety, and depression that might be associated with artificial joint awareness.

\section{CONCLUSION}

The ability to forget the artificial joint is associated with kinesiophobia and functional performance in patients with THA. This study shows that artificial joint awareness and high kinesiophobia develops in patients with THA and patients have a tendency to protect their artificial joints in long-term after surgery. Kinesiophobia and low adapting to artificial joint may cause impairment in functional performance accompanying with the limiting participation in daily living activities in the long term. Therefore, the artificial joint awareness should be taken into consideration while performing physiotherapy and rehabilitation to increase functional performance and to reduce kinesiophobia. We suggest a holistic approach aims to reduce the awareness of artificial joint should be added to the rehabilitation program of the patients with total hip arthroplasty.

\section{Acknowledgments}

The authors would like to thank all the patients and thank the Hacettepe University for providing equipment assistance for this study.

\section{REFERENCES}

[1] Gossec L, Paternotte S, Maillefert JF, Combescure C, Conaghan PG, Davis AM, Gunther KP, Hawker G, Hochberg M, Katz JN. The role of pain and functional impairment in the decision to recommend total joint replacement in hip and knee osteoarthritis: an international cross-sectional study of 1909 patients. Report of the OARSI-OMERACT Task Force on total joint replacement. Osteoarthritis Cartilage. 2011;19(2):147154.

[2] Davis A, Perruccio A, Ibrahim S, Hogg-Johnson S, Wong $R$, Streiner DL, Beaton DE, Cote P, Gignac MA, Flannery J. The trajectory of recovery and the inter-relationships of symptoms, activity and participation in the first year following total hip and knee replacement. Osteoarthritis Cartilage. 2011;19(12):1413-1421.

[3] Larsson A, Rolfson O, Kärrholm J. Evaluation of Forgotten Joint Score in total hip arthroplasty with Oxford Hip Score as reference standard. Acta Orthop. 2019:1-8.

[4] Hamilton D, Giesinger J, MacDonald D, Simpson A, Howie C, Giesinger K. Responsiveness and ceiling effects of the Forgotten Joint Score-12 following total hip arthroplasty. Bone Joint Res. 2016;5(3):87-91.

[5] Thienpont E, Opsomer G, Koninckx A, Houssiau F. Joint awareness in different types of knee arthroplasty evaluated with the Forgotten Joint score. J Arthroplasty. 2014;29(1):4851.

[6] Olver J. Do patient reported outcome measures in hip and knee arthroplasty rehabilitation have robust measurement attributes? A systematic review. J Rehabil Med. 2011;43(7):572583

[7] Suda AJ, Seeger JB, Bitsch RG, Krueger M, Clarius M. Are patients' expectations of hip and knee arthroplasty fulfilled? A prospective study of 130 patients. Orthop. 2010;33(2).

[8] Alviar MJ, Olver J, Brand C, Tropea J, Hale T, Pirpiris M, Khan F. Do patient-reported outcome measures in hip and knee arthroplasty rehabilitation have robust measurement attributes? A systematic review. J Rehabil Med. 2011;43(7):572583.

[9] Hamilton D, Loth F, Giesinger J, Giesinger K, MacDonald D, Patton JT, Simpson AH, Howie CR. Validation of the English language Forgotten Joint Score-12 as an outcome measure for total hip and knee arthroplasty in a British population. Bone Joint J. 2017;99(2):218-224.

[10] Suk M, Norvell DC, Hanson B, Dettori JR, Helfet D. Evidencebased orthopaedic surgery: what is evidence without the outcomes?. J Am Acad Orthop Surg. 2008;16(3):123-129.

[11] Wright RW. Knee injury outcomes measures. J Am Acad Orthop Surg. 2009;17(1):31-39.

[12] 12. Harris K, Dawson J, Gibbons E, Lim CR, Beard DJ, Fitzpatrick $R$, Price AJ. Systematic review of measurement properties of patient-reported outcome measures used in patients undergoing hip and knee arthroplasty. Patient Relat Outcome Meas. 2016;7:101-108. 
[13] Monticone M, Ferrante S, Rocca B, Salvaderi S, Fiorentini R, Restelli M, Foti Calogero et. Home-based functional exercises aimed at managing kinesiophobia contribute to improving disability and quality of life of patients undergoing total knee arthroplasty: a randomized controlled trial. Arch Phys Med Rehabil. 2013;94(2):231-239.

[14] Morgounovski J, Vuistiner P, Léger B, Luthi F. The fearavoidance model to predict return to work after an orthopedic trauma. Ann Phys Rehabil Med. 2016;59:110-111.

[15] Doury-Panchout F, Metivier J, Fouquet B. Kinesiophobia negatively influences recovery of joint function following total knee arthroplasty. Eur J Phys Rehabil Med. 2015;51(2):155161.

[16] Miki H, Sugano N, Hagio K, Nishii T, Kawakami H, Kakimoto A, Nakamura N, Yoshikawa H. Recovery of walking speed and symmetrical movement of the pelvis and lower extremity joints after unilateral THA. J Biomech. 2004;37(4):443-455.

[17] Sicard-Rosenbaum L, Light KE, Behrman AL. Gait, lower extremity strength, and self-assessed mobility after hip arthroplasty. The Journals of Gerontology Series A: Biol Sci Med Sci. 2002;57(1):47-51.

[18] Vogt L, Brettmann K, Pfeifer K, Banzer W. Walking patterns of hip arthroplasty patients: some observations on the mediolateral excursions of the trunk. Disabil Rehabil. 2003;25(7):309317.

[19] Van den Akker-Scheek I, Stevens M, Bulstra SK, Groothoff JW, van Horn JR, Zijlstra W. Recovery of gait after short-stay total hip arthroplasty. Arch Phys Med Rehabil. 2007;88(3):361-367.

[20] Van den Akker-Scheek I, Zijlstra W, Groothoff JW, Bulstra SK, Stevens M. Physical functioning before and after total hip arthroplasty: perception and performance. Phys Ther. 2008;88(6):712-719.

[21] Vissers M, Bussmann J, De Groot I, Verhaar J, Reijman M. Physical functioning four years after total hip and knee arthroplasty. Gait Posture. 2013;38(2):310-315.

[22] De Groot IB, Bussmann HJ, Stam HJ, Verhaar JA. Small increase of actual physical activity 6 months after total hip or knee arthroplasty. Clin Orthop Relat Res. 2008;466(9):2201-2208.

[23] Heiberg KE, Bruun-Olsen V, Ekeland A, Mengshoel AM. Effect of a walking skill training program in patients who have undergone total hip arthroplasty: followup one year after surgery. Arthritis Care Res. 2012;64(3):415-423.

[24] 24. Laupacis A, Bourne R, Rorabeck C, Feeny D, Wong C, Tugwell $P$, Leslie $K$, Bullas R. The effect of elective total hip replacement on health-related quality of life. J Bone Joint Surg Am. 1993;75(11):1619-1626.

[25] Behrend H, Giesinger K, Giesinger JM, Kuster MS. The "forgotten joint" as the ultimate goal in joint arthroplasty: validation of a new patient-reported outcome measure. J Arthroplasty. 2012;27(3):430-436.
[26] Kinikli Gi, Deniz HG, Karahan S, Yüksel E, Kalkan S, Kara D, Önal $S$, Sevinç C, Çağlar Ö, Atilla B. Validity and reliability of Turkish version of the Forgotten Joint Score-12. J Exerc Ther Rehabil. 2017;4(1):18-25.

[27] Yilmaz ÖT, Yakut Y, Uygur F, ULUĞ N. Tampa Kinezyofobi Ölçeği'nin Türkçe versiyonu ve test-tekrar test güvenirliği. Fizyoterapi Rehabilitasyon. 2011;22(1):44-49.

[28] Vlaeyen JW, Linton SJ. Fear-avoidance and its consequences in chronic musculoskeletal pain: a state of the art. Pain. 2000;85(3):317-332.

[29] Harada ND, Chiu V, Stewart AL. Mobility-related function in older adults: assessment with a 6-minute walk test. Arch Phys Med Rehabil. 1999;80(7):837-841.

[30] Enright PL, Sherrill DL. Reference equations for the sixminute walk in healthy adults. Am J Respir Crit Care Med. 1998;158(5):1384-1387.

[31] Thienpont E, Berghe AV, Schwab P-E, Forthomme JP, Cornu O. Joint awareness in osteoarthritis of the hip and knee evaluated with the 'Forgotten Joint'Score before and after joint replacement. Knee Surg Sports Traumatol Arthrosc. 2016;24(10):3346-3351.

[32] Matsumoto M, Baba T, Ochi H, Ozaki Y, Watari T, Homma Y, Kaneko K. Influence of the contralateral hip state after total hip arthroplasty on patient-reported outcomes measured with the forgotten joint score-12. Eur J Orthop Surg Traumatol. 2017;27(7):929-936.

[33] Kori S. Kinisophobia: a new view of chronic pain behavior. J Pain Manag. 1990:35-43.

[34] Sengul YS, Unver B, Karatosun V, Gunal I. Assessment of painrelated fear in patients with the thrust plate prosthesis (TPP): Due to hip fracture and hip osteoarthritis. Arch Gerontol Geriatr. 2011;53(2):249-252.

[35] Padovan AM, Kuvačić G, Gulotta F, Sellami M, Bruno C, Isoardi $M$, De Giorgia A. A new integrative approach to increase quality of life by reducing pain and fear of movement in patients undergoing total hip arthroplasty: the IARA model. Psychol Health Med. 2018;23(10):1223-1230.

[36] Temporiti F, Zanotti G, Furone R, Molinari S, Zago M, Loppini M, Galli M, Grappiola G, Gatti R. Gait analysis in patients after bilateral versus unilateral total hip arthroplasty. Gait Posture. 2019;72:46-50.

[37] Ahmad M, Xypnitos F, Giannoudis P. Measuring hip outcomes: common scales and checklists. Injury. 2011;42(3):259-264.

[38] Ashby E, Grocott M, Haddad F. Outcome measures for orthopaedic interventions on the hip. J Bone Joint Surg Br. 2008;90(5):545-549.

[39] Heiberg KE, Ekeland A, Bruun-Olsen V, Mengshoel AM. Recovery and prediction of physical functioning outcomes during the first year after total hip arthroplasty. Arch Phys Med Rehabil. 2013;94(7):1352-1359. 